\title{
Biomimetic organization of collagen matrices to template bone-like microstructures
}

\author{
Brian Wingender ${ }^{a}$, Patrick Bradley ${ }^{b}$, Neha Saxena ${ }^{a}$, Jeffrey W. Ruberti ${ }^{b *}$, Laurie Gower ${ }^{a^{*}}$ \\ a - Department of Materials Science and Engineering, University of Florida, Gainesville, FL \\ $\mathrm{b}$ - Department of Bioengineering, Northeastern University, Boston, MA \\ *Corresponding Author(s) - LG (lgower@mse.ufl.edu) and/or JR (j.ruberti@ neu.edu)
}

\begin{abstract}
The mineralized extracellular matrix (ECM) of bone is essential in vertebrates to provide structure, locomotion, and protect vital organs, while also acting as a calcium and phosphate reservoir to maintain homeostasis. Bone's structure comprises mainly structural collagen fibrils, hydroxyapatite nanocrystals and water, and it is the organization of the densely-packed collagen matrix that directs the organization of the mineral crystallites. Biogenic mineralization occurs when osteoblasts release "mineral bearing globules" which fuse into the preformed collagen matrix, and upon crystallization of this amorphous precursor, the fibrils become embedded with [001] oriented nanocrystals of hydroxyapatite. Our prior work has shown that this nanostructured organization of bone can be reproduced in vitro using the polymer-induced liquid-precursor (PILP) process. In this report, our focus is on using biomimetic processing to recreate both the nano- and micro-structure of lamellar bone. We first applied molecular crowding techniques to acidic, type-I collagen solutions to form dense, liquid crystalline collagen (LCC) scaffolds with cholesteric order. We subsequently mineralized these LCCs via the PILP process to achieve a high degree of intrafibrillar mineral, with compositions and organization similar to that of native bone and with a "lamellar" microstructure generated by the twisting LCC template. In depth characterization of the nano- and micro-structure was performed, including optical and electron microscopy, X-ray and electron diffraction, and thermogravimetric analyses. The results of this work lead us closer to our goal of developing hierarchically structured, collagen-hydroxyapatite composites which can serve as fully synthetic, bioresorbable, load-bearing bone substitutes that are remodeled by the native BRU.
\end{abstract}

\begin{abstract}
Abbreviations
ECM, extracellular matrix; BRU, bone remodeling unit; LCC, liquid crystalline collagen; PEG, polyethylene glycol; PILP, polymer-induced liquid-precursor; pAsp, poly-L-aspartic acid sodium salt; TBS, Tris buffered saline; TEM, transmission electron microscope; SAED, selected area electron diffraction; SEM, scanning electron microscope; WAXD, wide angle X-ray diffraction; TGA, thermogravimetric analysis; ACP, amorphous calcium phosphate; $\mathrm{CaP}$, calcium phosphate
\end{abstract}

\section{Keywords}

Collagen; hydroxyapatite; PILP; cholesteric; amorphous calcium phosphate; biomimetic bone 


\section{Introduction}

The extracellular matrix (ECM) of bone is a dynamic environment which serves multiple functions, ranging from mechanical roles such as protection of vital organs and providing a means for locomotion and stability, to metabolic functions acting as an ionic reservoir to help maintain homeostasis [1]. Despite decades of research, bone related diseases (e.g. osteoporosis, osteogenesis imperfecta, etc.) and injuries remain a significant clinical challenge and exacerbate the need for a synthetic, bioresorbable substitute [2-4]. Unfortunately, bone morbidities account for half of all chronic conditions worldwide in people over the age of 50, and this is expected to increase with global growth of an ageing population [5-7]. Many researchers are investigating composite-based biomaterials for potential use as bone grafts due to the demanding requirements of scaffold design for bone regenerative engineering. However, the lack of current viable options for the treatment of load-bearing, critical-sized bone defects illustrates the need for novel, synthetic materials where mechanical integrity of the scaffold must be maintained during bioresorption and replacement with host tissue [5]. The use of hierarchical, collagenhydroxyapatite composites as biocompatible scaffolds for tissue regeneration strategies seems a promising approach due to their osteoconductivity, cell compatibility, bioresorptivity, and synergistic mechanical properties [8-14]. Towards the long-term goal of preparing a bioresorbable, load-bearing bone substitute, a crucial first step is the development of a bulk scaffold material with a dense, bone-like organization and composition. Our biomimetic approach to achieve this goal is to emulate the natural processes of bone formation to create a material with the nano- and micro-structure of lamellar bone, which we anticipate will provide mechanical properties and resorption potential of native bone.

\subsection{The composition and organization of bone extracellular matrix}

Bone is composed primarily of type I collagen protein ( 20 wt $\%)$, highly substituted hydroxyapatite mineral ( $65 \mathrm{wt} \%)$, water $(\sim 10 \mathrm{wt} \%)$, and various non-collagenous proteins (NCPs, $\sim 5 \mathrm{wt} \%$ ); the latter of which are believed to play important roles in the biomineralization process $[15,16]$ (see [17] for a detailed discussion). Collagen fibrils are composed of a periodic arrangement of assembled tropocollagen, which results in a characteristic $67 \mathrm{~nm}$ banding periodicity, comprising the gap and overlap zones [18]. Load-bearing tissues commonly have fibrils organized in parallel arrays, which then are arranged into array patterns at the next level of hierarchy [19]. Lamellar bone has a mesoscale arrangement where adjacent lamellae of dense, parallel fibril arrays have offset orientations between neighboring layers. The thickness and rotational offset of lamellae can vary with anatomical location and from species to species [19], but is generally on the order of a few microns. This microstructural arrangement is often referred to as a 'rotated-plywood' motif, and has been suggested to arise from the cholesteric liquid crystalline order that can result from concentrated solutions of collagen [20,21]. Soon after osteoid deposition, the process of biomineralization begins as the collagen is infiltrated with "mineral bearing globules" that are secreted by osteoblasts into the extracellular milieu [22, 23].

The mineralized collagen fibril is considered to be a basic building block of mineralized tissue. The morphology of hydroxyapatite in bone has often been described as thin, irregular, plateletshaped crystals with average dimensions of $50 \times 25 \times 5 \mathrm{~nm}$ [19]. The crystallographic $c$-axis of the mineral is aligned with the fibril axis producing an interpenetrating, organic-inorganic nanocomposite with remarkable mechanical properties [24, 25]. Throughout an organism's lifetime, bone remodeling units (BRU), also referred to as basic multicellular units (BMU), work to resorb old bone and replace it with new (unmineralized) collagenous osteoid [26-28] to maintain mechanical integrity and repair damaged bone [29]. The interpenetrating, composite 
organization of bone has important implications for its bioresorptive potential, as well as the overall mechanical properties of bone (and bone substitutes) across multiple length scales.

\subsection{Biomimetic processing and structures for bone tissue engineering}

Most of the current knowledge regarding collagen self-assembly is a result of in vitro studies in which fibrils are reconstituted from purified type-I collagen isolated from bovine tendon and skin [30]. Densification of dilute collagen solutions has enabled researchers to generate a variety of collagen scaffolds with control over parameters such as final concentration, homogeneity, and shape [31-34]. Giraud-Guille and colleagues conducted experiments which formed liquid crystalline, or cholesteric, collagen phases by densifying acidic collagen solutions beyond a critical concentration $(\sim 90 \mathrm{mg} / \mathrm{ml})$ and stabilizing the organization through fibrillogenesis induced via neutralization by ammonia vapor diffusion [31, 34-39]. Here we use a similar molecular crowding/confining mechanism to generate dense, liquid crystalline collagen (LCC) scaffolds. However, by permitting the system to equilibrate with a neutral poly(ethylene glycol) (PEG) solution placed outside a dialysis cassette, which also acts as an osmicant to concentrate the solution, fibrillogenesis can be induced while avoiding cytotoxicity associated with ammonia $[32,33]$. Additionally, by selecting a PEG concentration which balances the osmotic pressure across the dialysis membrane, densification proceeds in a manner that eliminates heterogeneities associated with the dual interface method (i.e. combined evaporation/dialysis). In both the Giraud-Guille and Ruberti systems, the processing is not fully biomimetic because the densification reaction in the biological realm consists of enzymatic cleavage to produce tropocollagen, which is neither easy nor affordable in the beaker; but the outcome is a biomimetic structure of densely-packed collagen scaffolds with similar cholesteric order.

With respect to the biomineralization reaction, previous work from our group has shown that the polymer-induced liquid precursor (PILP) process is capable of reproducing the fundamental nanostructure of bone. Recently, in vivo observations have shown evidence of amorphous calcium phosphate (ACP) phases involved in the mineralization of zebrafish caudal fin arrays $[22,23]$. The ACP phase appears to be transported to the pre-formed collagen matrix by secretion of cellular vesicles that contain "mineral-bearing globules", which fuse to and infiltrate the collagen matrix. The PILP process is a biomimetic in vitro mineralization model in which acidic polypeptides, used as synthetic analogues of the NCP's present in bone, stabilize a liquidlike amorphous mineral precursor that is also able to penetrate into dense collagen scaffolds and infiltrate the individual collagen fibrils. The intrafibrillar hydroxyapatite crystals become embedded with their [001] crystallographic axes well-aligned with the fibril axes [40-44]. In vitro precursor-based mineralization models, reviewed by Nudelman et al., have generally tended toward the mineralization of individual collagen fibrils, porous collagen sponges, or demineralized native tissue scaffolds using a variety of natural and synthetic proteins/polypeptides as process directing agents [45-47]. Our previous work was focused on optimizing the PILP processing parameters to achieve intra- and extra-fibrillar mineralization of collagen sponges, to create materials with a bone-like nanostructure and composition. While these model systems have provided valuable information in identifying new physico-chemical mechanisms that can lead to collagen mineralization, such low density scaffolds are highly porous and do not provide load-bearing properties. Therefore, we are now focused on methods to provide densely-packed collagen arrays, which emulate the microstructural organization of lamellar bone, to serve as scaffolds for biomimetic mineralization. 


\section{Results and Discussion}

In this study, we have combined two biomimetic strategies from our respective labs: we initially prepared dense LCC scaffolds developed by Ruberti's group [33], which were then mineralized via the PILP process developed by Gower's group [48]. First, we will provide an overview of the resulting composites to demonstrate that LCC scaffolds can be mineralized by the PILP process. Then the following two sections will delve into more detail by characterizing the resulting structures from each processing step to show that the organized LCC scaffolds can serve as passive templates for creating composites that have hydroxyapatite nanocrystals embedded within a fibrillar, collagen matrix.

The LCC scaffolds, which have densities of $\sim 250 \mathrm{mg} / \mathrm{ml}$, are relatively thin $(\sim 50 \mu \mathrm{m})$, so they are initially semi-transparent and flexible (Fig. 1A); but then become stiff and opaque after mineralization (Fig. 1B). When mechanically fractured and examined in SEM, the interior of the scaffolds appears to be aligned over large distances. Although mineral crystals are not seen in SEM, EDS shows the presence of large calcium and phosphate peaks (Fig. 1C) which indicates that the majority of the mineral is inside the fibrils given that crystallites cannot be seen on the surface. The nanostructural organization in ultrathin cross-sections, observed via TEM, revealed highly aligned collagen fibrils with characteristic D-periodicity (enhanced by heavy metal staining) in unmineralized LCC scaffolds (Fig. 1D). Staining of mineralized sections was not required in TEM as the electron dense mineral phase provides strong Z-contrast (Fig. 1E). The mineral phase was determined to be hydroxyapatite via XRD (Fig. 1F), and a high degree of mineralization (64-72 wt\%) was measured via TGA (Fig. 1G), confirming the high mineral content suggested by the large EDS peaks.

\subsection{Generation of dense lamellar collagen scaffolds via liquid crystal processing}

Polarized light microscopy revealed strong birefringent domains throughout the sample due to the cholesteric ordering of the highly concentrated and aligned collagen (Fig. 2A). These patterns were consistent with the banded cords $(*)$, zig-zag extinction patterns (double-headed arrow), and cholesteric organization of dense collagen scaffolds cited in the literature $[31,34,36]$. These patterns result from light that is transmitted through the sample when the collagen fibrils are oriented with their axes in the preparation plane, diminished when oblique, and extinguished when perpendicular (Fig 2A inset). When examined with SEM, the samples appeared to have highly aligned, fibrillar collagen along with elongated pore structures (Fig. 2B). These pores are likely artifacts of sample preparation due to ice crystal growth that occurs in unfixed samples during the flash-freezing process prior to lyophilization. Indeed, TEM observations of fixed ultrathin sections at low magnification reveal dense packing and aligned fibrils, with no distinguishable pore space (Fig. 2C). When brightness and contrast were altered, the characteristic D-banding stands out on fibrils with their axes oriented in the plane of the section, while fibrils with axes orthogonal to the section appear end-on as round shapes (Fig. 2D). Heavy metal staining was also used to enhance contrast in TEM to help visualize the collagen banding in other domains that were present, such as aligned and isotropic (Fig. 2E and 2F, respectively), according to the terminology applied by Wang et al. [49]. Interestingly, in many areas the Dbanding pattern was in register across multiple fibrils (Fig. $2 \mathrm{E}$ and F), suggesting well-aligned tropocollagen can provide continuity prior to fibrillogenesis. Fibril diameters were difficult to measure due to scaffold density and overlapping of fibrils in prepared TEM sections, however average D-periodicity, measured with FIJI (ImageJ, NIH) statistical software, was $64 \pm 4 \mathrm{~nm}$, which is in good agreement with measurements reported in the literature [50]. 


\subsection{Mineralization of collagen via the polymer-induced liquid-precursor (PILP) process}

Dense collagen films were mineralized for up to two weeks in Tris buffered saline solution supersaturated with calcium and phosphate, using poly(aspartic acid) (pAsp, $27 \mathrm{kDa}$ ) as the PILP process-directing agent $[42,48,51]$. After mineralization, the collagen films were qualitatively much more stiff and brittle than before mineralization, and had a white, opaque appearance. In SEM, aside from a few large particles on the surface, there was no visible evidence of extrafibrillar mineralization even at higher magnifications (inset, top), yet the EDS (inset, bottom) analysis indicated the presence of large amounts of calcium and phosphorous from intrafibrillar mineral (Fig. 3A). In the interior of a mechanically freeze-fractured sample, a gradual shift of orientation can be seen by the rotation of aligned collagen fibrils, which is suggestive of cholesteric order, and this creates adjacent lamellae that appear to have given rise to the layered fracture surface (Fig. 3B). Cryo-pulverized specimens showed individual mineral crystals that exhibited a platelet morphology which, when viewed edge-on, appeared dark and needle-like (arrows; Fig. 3C), closely resembling mineral extracted from native bone [19]. The shifting appearance of platelets to needles can be observed nicely in the tomographic video shown in the Supplementary Information (Vid. S1). Some fragments consisted of one or two mineralized fibrils containing intrafibrillar crystals well-aligned with the fibril axis, and also appeared to have extrafibrillar mineral crystals which were not as well co-oriented (Fig. 3D), leading to a less defined fibril surface (relative to our prior studies using collagen sponges). Larger fragments were difficult to image in TEM due to their thickness, but some contained multiple aligned fibrils which exhibited dark bands, and given the spacing of $\sim 67 \mathrm{~nm}$ (Fig. 3E) matches that of native collagen, it helps to identify the fibril orientation. The electron dense mineral is presumably infiltrating the gap zones, but it not restricted to these spaces, because mineral can be seen to spread and crystallize throughout the intrafibrillar collagen nanocompartments [52]. As the mineralization proceeds and crystallization occurs, the increasing electron density (and dimensions) of the mineral phase masks the banding of the collagen fibrils in TEM. Nevertheless, at the thinner edge, one can see many dark striations from crystals that align parallel to the fibril, yet traverse much further than the gap zones. Nudelman et al. showed with cryo-EM that "amorphous $\mathrm{CaP}$ nanocomplexes" infiltrate well-formed fibrils at the gap zones, where they also appear to spread throughout the collagen interstices prior to crystallization [53].

In bright-field TEM, Figs. 4A and B show highly mineralized regions that resemble native bone sections from the literature $[19,54]$, where it is often difficult to isolate individual fibrils when they are densely packed. The circles in A and B represent the region of interest chosen with the selected area aperture to create the corresponding diffraction patterns, which show arcs from the (002) and (004), indicating that the [00l] $c$-axis of the hydroxyapatite crystals is generally aligned with the fibril axis. In dark-field TEM, a specific beam(s) from the diffraction pattern is selected with a pinhole aperture to create the image so that only the crystals contributing to the selected beam appear bright against a dark background (Fig. 4. E and F). To create the dark-field image in Fig. 4E, the entire (002) arc was selected from the diffraction pattern (red circle, Fig. 4C) generated from the selected area shown in the white circle of Fig. 4A. Conversely, for the dark-field image in Fig. 4F, a smaller pinhole aperture was used to select just the (002) spot indicated by the red circle in Fig. 4D, such that only the crystals in that specific (near vertical) orientation contribute to the dark field image, even though the entire region is highly mineralized. In both examples, the dimensions are large enough to contain several collagen fibrils, and the organization of the mineral appears to track along with the aligned collagen, although the fibrils often weave in and out of the ultrathin section plane, making it difficult to follow for long distances. 


\subsection{Composition of PILP-mineralized LCC}

Microscopy was used to investigate the hierarchical structure of the scaffolds before and after mineralization. The PILP process resulted in both intrafibrillar and extrafibrillar mineralization of the collagen scaffold, and the mineral phase was determined to be hydroxyapatite via wideangle $x$-ray diffraction (WAXD). WAXD patterns of the PILP-mineralized LCC scaffolds closely resemble that of bovine cortical bone, with typical peaks for HA appearing at $26^{\circ}$ and $32^{\circ}$ (Fig. 5). In this sample of mature bone, the peaks are sharp and narrow because the mineral has grown larger and become more crystalline compared with those of the PILP-mineralized collagen, which exhibit broad and less intense peaks due to either small crystallite size and/or the presence of amorphous or poorly crystalline calcium phosphate. It should be noted that the peak at $26^{\circ}$ is less prominent in the PILP-mineralized samples because there may be preferential orientation of those crystal planes within the well-aligned scaffold, as seen in our prior studies with highly oriented turkey tendons [40]. The peak occurring at $\sim 39^{\circ}$ for PLCC2 and PLCC3 can be attributed to diffraction from the (310) plane, and is exaggerated in this sample by collagen fibril orientation with respect to the incident X-ray (in this same orientation the peaks from the (00l) planes, at $26.5^{\circ}$ and $\sim 53^{\circ}$, are diminished) [40]). Thermal analysis of the PILP-mineralized LCC showed a high degree of mineralization was achieved (64-72 wt\%), where mineral content was determined by residual ash weight measurements (Fig. 6). Overall, the biomimetic composite closely matches native lamellar bone in composition, hierarchy, and organization across multiple length scales.

\subsection{Implications for use in hard tissue engineering or as a bone model system}

As mentioned earlier, a hurdle to overcome with the biomimetic approach is the limited depth of mineral penetration into bulk scaffolds. This was even the case in the non-biomimetic approach used by Wang et al. [49], where their one-step co-precipitation/fibrillogenesis method did lead to a macroscale scaffold, yet the overall mineral content was only $20 \mathrm{wt}$. \% (according to their TGA data in the Supplementary Information Fig. S3). Our collagen-hydroxyapatite film was developed to ultimately assemble multiple films into a laminated composite, which will emulate lamellar bone at the macroscale, with the long-term goal of making a bulk, bioresorbable bone substitute which can maintain a load-bearing capacity as it is remodeled by the BRU. This is in contrast to many approaches that make biodegradable scaffolds, where the degradation rate needs to closely match that of the infiltration of new bone. Biodegradable composites which break down via hydrolytic activity require customization because degradation rates can be highly variable amongst patients or at different locations within the same patient. Therefore, we anticipate that it might be a distinct advantage to have resorption of the implant be regulated through the concerted action of the BRU, as occurs during natural bone remodeling, where osteoblasts lay down new bone in synchrony with the osteoclasts removal of old bone. To accomplish this with a synthetic material, the cells need to "see" a structure that resembles bone nanostructure and contains the appropriate ligands to trigger cell attachment and activity. We have shown this capability of osteoclast resorption with PILP-remineralized bone in our prior study, and found a pronounced impact when using osteopontin as the PILP process-directing agent [44]. Our next step is to use these dense collagen matrices to further examine such bioactivity issues with a fully synthetic scaffold, where bioactive factors can be regulated and tailored to examine cell interactions.

There are still many unknown aspects regarding the formation of bone and the metabolic modeling and remodeling events that continue throughout life [27]. The recent advances of genetic knockout animals has enabled researchers to probe questions about biomineralization in the absence of certain proteins. Due to the large number of NCP's present during 
biomineralization, and the seemingly functional redundancy amongst them, it is difficult to ascertain the role of individual remaining proteins in these models [55]. Therefore, we believe that the lack of biocomponents in this collagen/hydroxyapatite composite make it an interesting candidate for in vitro studies as a clean, purely synthetic "knock-in" model system. This system would allow for osteoinductive or other bioactive components (e.g. NCP's, growth factors, proteoglycans, etc.) to be selectively incorporated into the collagen scaffold, via bi-molecular crowding strategies [33], which would enable researchers to examine their individual effects on cellular response in an in vitro model system. Furthermore, this system may provide a means to study the BRU (such as the influence of NCP's on osteoclast activation) in a more controlled 3D environment that better emulates bone's composition and hierarchical structure (compared to current studies using 2D models) at length scales appreciable to cells. This would reduce the need for animal sacrifice while providing complementary data to current "knock-out" mouse models.

\section{Conclusion}

Molecular crowding techniques were used to densify acidic, type-I collagen solutions, driving the tropocollagen into cholesteric arrangements which were stabilized by raising the $\mathrm{pH}$ to induce fibrillogenesis. These dense, organized collagen scaffolds were then successfully mineralized via the PILP process, leading to hierarchical composites with a high degree of mineralization and composition matching that of lamellar bone. Hydroxyapatite crystals were embedded within the collagen fibrils and [001] oriented along the $c$-axis of the fibrils, which mimicked the nanoscale organization of a mineralized native fibril. At the microstructural level, parallel arrays of banded collagen fibrils could be seen extending over several microns in unmineralized sections, in which the cholesteric twisting led to a lamellar type of microstructure. Current studies are directed at determining the differences that process-directing agents have on the extent of mineralization, measuring the material's mechanical properties, as well as feasibility of osteoclastic resorption of synthetic biomimetic bone composites.

\section{Materials and Methods}

\subsection{Molecular Crowding: Liquid Crystalline Collagen (LCC) Scaffold}

Molecular crowding of collagen was achieved by concentrating type I collagen molecules (TELOCOL®, Advanced Biomatrix) to densities found in the loadbearing tissues and beyond (100-400 mg/mL), as described by [33]. Briefly, concentrated collagen (i.e. crowded collagen) was produced by dialyzing $\sim 3 \mathrm{mg} / \mathrm{mL}$ acetic acid solution of type I collagen monomers against acidic polyethylene glycol (PEG, 35k MW, 40\% wt./vol., $\mathrm{pH} \sim 2.5$ via $\mathrm{HCl}$ titration, SigmaAldrich, St. Louis, MO) at $4{ }^{\circ} \mathrm{C}$ in a $10 \mathrm{~mL}$ dialysis cassette (Slide-A-Lyzer ${ }^{\mathrm{TM}} 3.5 \mathrm{k} \mathrm{MWCO}$, ThermoFisher Scientific) for 1 week. At this point, the dialysis cassette was removed and submerged in neutralized PEG (40\% wt/vol, $\mathrm{pH} \sim 7.4)$ and allowed to equilibrate for 1 week to induce fibrillogenesis, then transferred to an incubator for 4 hours $\left(37^{\circ} \mathrm{C}, 100 \%\right.$ relative humidity) to further polymerize the scaffold. The LCC scaffolds were then carefully cut out, removed from the dialysis membrane with tweezers, and thoroughly rinsed by immersion in $1 \mathrm{X}$ PBS for 15 mins at $20^{\circ} \mathrm{C}$. Half of each scaffold was kept for ultrastructural characterization and the other half was mineralized.

\subsection{Mineralization of LCC Scaffold}

The mineralization solution was prepared by mixing equal volumes of $9 \mathrm{mM} \mathrm{CaCl}_{2} \cdot 2 \mathrm{H}_{2} \mathrm{O}$

(Sigma, St. Louis, MO) and $4.2 \mathrm{mM} \mathrm{K}_{2} \mathrm{HPO}_{4}$ (Sigma, St. Louis, MO) solutions, chosen based on 
successful results from our prior studies $[41,48,51]$. To maintain the $\mathrm{pH}$ of the mineralization solution at 7.4, calcium and phosphate solutions were made in Tris-buffered saline (TBS) containing $0.9 \%(\mathrm{w} / \mathrm{v}) \mathrm{NaCl}$ and $0.02 \%$ (w/v) sodium azide (Sigma, St. Louis, MO) to prevent microbial activity. The process-directing agent, poly-L-aspartic acid (27kDa, Alamanda Polymers, Inc.), was added at a concentration of $50 \mu \mathrm{g} / \mathrm{ml}$ to $250 \mathrm{~mL}$ of the calcium solution before mixing with an equal volume of the phosphate counterion solution. An additional control group was made where the mineralization solution contained no polymeric additive. The LCC scaffolds were incubated in the mineralization solution under vacuum conditions for 30 min to remove any air bubbles. After degassing the sample, the mineralization reaction was kept in an oven at $37{ }^{\circ} \mathrm{C}$ to emulate physiological conditions under gentle stirring (stir bar $100 \mathrm{rpm}$ ) for up to 2 weeks. At 7 and 14 days, the mineralized samples were removed from the solution, copiously washed with DI water ( $3 \mathrm{x}$ for $15 \mathrm{mins}$ ), lyophilized and stored at $20^{\circ} \mathrm{C}$ until further use, or embedded in resin for characterization.

\subsection{Scanning Electron Microscopy with Energy Dispersive Spectroscopy}

Surface- and cross-sections of mineralized samples were prepared for examination by the manual freeze-fracture technique adapted from [51]. The sections were mounted on aluminum stubs with double-sided copper tape and sputter coated twice with amorphous carbon to enhance conductivity. The microscale organization of mineralized samples was then observed using a 6400 JEOL SEM instrument, equipped with an energy dispersive spectrometer, at an accelerating voltage of $15 \mathrm{kV}$. For elemental analysis of mineralized samples, EDS analysis was done during SEM examination.

\subsection{Resin Embedding and Ultramicrotomy}

Small strips (approximately $3 \mathrm{~mm} \times 10 \mathrm{~m}$ x $50 \mu \mathrm{m}$ ) of unmineralized and mineralized LCC were carefully cut from the bulk sample and fixed overnight, at room temperature, with $4 \%$ paraformaldehyde in 1X PBS. Samples were then dehydrated through a series of graded ethanol and embedded in acrylic resin (LR White Hard, Electron Microscopy Sciences) for sectioning. Ultrathin sections $(\sim 90 \mathrm{~nm})$ were cut on a Leica Ultracut $\mathrm{T}$, at a rate of $8 \mathrm{~mm} / \mathrm{s}$, using a diamond knife and floated on double-distilled water. Sections were collected on carbon-coated 200 mesh copper TEM grids for TEM analysis. Unmineralized LCC samples were stained with heavy metal salts (2\% uranyl acetate aq.) to increase contrast.

\subsection{Polarized Light Microscopy}

Semi-thin sections $(\sim 1 \mu \mathrm{m})$, traverse to the sample surface, of unmineralized LCC scaffolds were prepared via ultramicrotome as described above. Floating sections were lifted out of the diamond knife boat, transferred to a glass slide then air dried. Sections were observed with an Olympus BX60 microscope between crossed-polars. Molecular orientations were visualized by the use of a gypsum first-order red retardation plate inserted at $45^{\circ}$ between the polarizer and analyzer [36]. Images were captured with a Lumenera Infinity 3 digital camera.

\subsection{Liquid Nitrogen Freezing and Mechanical Pulverization}

A small amount $(\sim 10 \mathrm{mg})$ of PILP mineralized LCC was submerged in liquid nitrogen and mechanically pulverized using a mortar and pestle. The pulverized sample was then suspended in a small amount of ethanol to be dropped onto a TEM grid. Copper 200 mesh, formvar coated TEM grids were placed on filter paper to absorb excess liquid, and three to five $7 \mu l$ drops were placed on each grid. Grids were allowed to air dry before examination in TEM. 


\subsection{Transmission Electron Microscopy and Selected Area Electron Diffraction}

Ultrathin sections were imaged with a Hitachi 7600 TEM (Hitachi High-Technologies America, Schaumburg, IL) equipped with a Macrofire monochrome progressive scan CCD camera (Optronics, Goleta, CA) and AMT image capture software version 600.335p (Advanced Microscopy Techniques, Danvers, MA) at an accelerating voltage of $80 \mathrm{kV}$ in bright field mode. To help visualize the crystallographic orientation of the hydroxyapatite crystals, ultrathin sections were imaged in bright field and SAED (dark field) modes were using a JEOL 2010F operating at $200 \mathrm{kV}$. Pulverized specimens were imaged with a JEOL 200CX operating at $200 \mathrm{kV}$.

\subsection{Wide-angle X-ray Diffraction}

WAXD analysis was used to determine the crystallographic structure of the samples. In order to observe the major HA peaks, which develop at around $26^{\circ}(002)$ and $32^{\circ}$ (combination of (211), (112) and (300)), the samples were scanned with $\mathrm{Cu} \mathrm{K \alpha}$ X-ray radiation from a Philips XRD ADP 3720 diffractometer at $40 \mathrm{kV}$ and $45 \mathrm{~mA}$, using a step size of $0.01^{\circ} \mathrm{mrad} \mathrm{s}^{-1}$ with a time of $10 \mathrm{~s} \mathrm{step}^{-1}$, over a $2 \theta$ range of $10-60^{\circ}$.

\subsection{Thermogravimetric Analysis}

To determine the degree of mineralization of the samples, thermogravimetric analysis was conducted using a TG/DTA 320 (Seiko, Thermo Haake, Germany) instrument. A heating rate of $20{ }^{\circ} \mathrm{C} \mathrm{min}{ }^{-1}$ was applied in the temperature range $30-600{ }^{\circ} \mathrm{C}$ under nitrogen at a flow rate of 100 $\mathrm{ml} \mathrm{min}{ }^{-1}$. To compare the degree of mineralization, the material remaining at $600{ }^{\circ} \mathrm{C}$ was interpreted as the mineral content since the organic portion of the samples should be totally combusted by $600{ }^{\circ} \mathrm{C}$ in a nitrogen-containing environment.

\section{Author Contributions}

LG and JR designed the project, PB and BW performed experiments. BW and NS collected and analyzed the data. BW wrote the paper. All authors read, contributed to discussion and approved the final manuscript.

\section{Acknowledgements}

This material is based upon work supported by the National Science Foundation under grant No. DMR-1309657. We would like to thank Dr. Douglas Rodriguez, University of Florida, and the use of the Macromolecular Center, for conducting the thermogravimetric analysis. We would also like to thank Dr. Sharon W. Matthews, University of Florida, for her training and expertise in sample preparation, microtomy, and microscopy. Data was generated using instruments from the Major Analytical Instrumentation Center and the College of Medicine Electron Microscopy Core Facility at the University of Florida. Any opinions, findings and conclusions or recommendations expressed in this material are those of the author(s) and do not necessarily reflect those of the National Science Foundation. 
References Cited

[1] Hancox NM. Biology of Bone: University Press; (1972).

[2] Laurencin C, Khan Y, El-Amin SF. Bone graft substitutes. Expert Review of Medical Devices

(2006);3:49-57.

[3] Greenwald AS, Boden SD, Goldberg VM, Khan Y, Laurencin CT, Rosier RN. Bone-Graft

Substitutes: Facts, Fictions, and Applications. The Journal of Bone \& Joint Surgery (2001);83:S98103.

[4] Bajaj P, Schweller RM, Khademhosseini A, West JL, Bashir R. 3D Biofabrication Strategies for

Tissue Engineering and Regenerative Medicine. Annual Review of Biomedical Engineering

(2014);16:247-76.

[5] Yu X, Tang X, Gohil SV, Laurencin CT. Biomaterials for Bone Regenerative Engineering.

Advanced Healthcare Materials (2015);4:1268-85.

[6] Baroli B. From natural bone grafts to tissue engineering therapeutics: Brainstorming on pharmaceutical formulative requirements and challenges. Journal of Pharmaceutical Sciences (2009);98:1317-75.

[7] Brinker MR, O'Connor DP. The Incidence of Fractures and Dislocations Referred for Orthopaedic Services in a Capitated Population. The Journal of Bone \& Joint Surgery (2004);86:2907.

[8] Bose S, Vahabzadeh S, Bandyopadhyay A. Bone tissue engineering using 3D printing. Materials Today (2013);16:496-504.

[9] Lee EJ, Kasper FK, Mikos AG. Biomaterials for Tissue Engineering. Annals of Biomedical Engineering (2014);42:323-37.

[10] Nair LS, Laurencin CT. Biodegradable polymers as biomaterials. Progress in Polymer Science (2007);32:762-98.

[11] Oryan A, Alidadi S, Moshiri A, Maffulli N. Bone regenerative medicine: classic options, novel strategies, and future directions. J Orthop Surg Res 2014 Mar 17;9(1):18 doi: 101186/1749-799X-9-18 (2014).

[12] Janicki P, Schmidmaier G. What should be the characteristics of the ideal bone graft substitute? Combining scaffolds with growth factors and/or stem cells. Injury (2011);42 Suppl 2:S77-81.

[13] Kanczler J. Osteogenesis and Angiogenesis: The potential for engineering bone. eCM Journal (2008);15:100-14.

[14] García-Gareta E, Coathup MJ, Blunn GW. Osteoinduction of bone grafting materials for bone repair and regeneration. Bone (2015);81:112-21.

[15] Boskey AL. Noncollagenous matrix proteins and their role in mineralization. Bone and Mineral (1989);6:111-23.

[16] Weiner S, Wagner HD. THE MATERIAL BONE: Structure-Mechanical Function Relations. Annual Review of Materials Science (1998);28:271-98.

[17] Gower LB. Biomimetic mineralization of collagen. In: Biomineralisation of Biomaterials:

Fundamentals and Applications. . Cambridge UK(2016).

[18] Chapman JA, Tzaphlidou M, Meek KM, Kadler KE. The collagen fibril--a model system for studying the staining and fixation of a protein. Electron Microsc Rev 1990;3(1):143-82 (1990).

[19] Weiner S, Traub W, Wagner HD. Lamellar Bone: Structureâ€“Function Relations. Journal of Structural Biology (1999);126:241-55.

[20] Gebhardt W. Uber funktionell wichtige Anordnungsweisen der eineren and groberen

Bauelemente des Wirbeltierkmochens.II. Spezieller Teil Der Bau der Haversschen

Lamellensysteme and seine funktionelle Bedeutung. Arch Entwickl Mech Org (1906);20:187-322.

[21] Giraud-Guille MM. Twisted plywood architecture of collagen fibrils in human compact bone osteons. Calcified Tissue International (1988);42:167-80.

[22] Mahamid J, Aichmayer B, Shimoni E, Ziblat R, Li C, Siegel S, et al. Mapping amorphous calcium phosphate transformation into crystalline mineral from the cell to the bone in zebrafish fin rays. Proc Natl Acad Sci U S A (2010);107:6316-21. 
[23] Mahamid J, Sharir A, Addadi L, Weiner S. Amorphous calcium phosphate is a major component of the forming fin bones of zebrafish: Indications for an amorphous precursor phase. Proc Natl Acad Sci U S A (2008);105:12748-53.

[24] Rho J-Y, Kuhn-Spearing L, Zioupos P. Mechanical properties and the hierarchical structure of bone. Medical Engineering \& Physics (1998);20:92-102.

[25] Currey J. Incompatible mechanical properties in compact bone. J Theor Biol 2004 Dec

21;231(4):569-80 (2004).

[26] Frost H. Tetracycline-based histological analysis of bone remodeling. Calcified Tissue Research (1969);3:211-37.

[27] Sims NA, Martin TJ. Coupling the activities of bone formation and resorption: a multitude of signals within the basic multicellular unit. BoneKEy Rep (2014);3.

[28] Clarke B. Normal Bone Anatomy and Physiology. Clinical Journal of the American Society of Nephrology : CJASN (2008);3:S131-S9.

[29] Hadjidakis DJ, Androulakis II. Bone Remodeling. Annals of the New York Academy of Sciences (2006);1092:385-96.

[30] Kadler KE, Holmes DF, Trotter JA, Chapman JA. Collagen fibril formation. Biochem J 1996 May 15;316 ( Pt 1):1-11.

[31] Wang Y, Silvent J, Robin M, Babonneau F, Meddahi-Pelle A, Nassif N, et al. Controlled collagen assembly to build dense tissue-like materials for tissue engineering. Soft Matter (2011);7:9659-64.

[32] Ruberti JW, Zieske JD. Prelude to corneal tissue engineering - gaining control of collagen organization. Prog Retin Eye Res 2008 Sep;27(5):549-77 doi: 101016/jpreteyeres200808001 Epub 2008 Aug 19 (2008).

[33] Saeidi N, Karmelek KP, Paten JA, Zareian R, DiMasi E, Ruberti JW. Molecular crowding of collagen: a pathway to produce highly-organized collagenous structures. Biomaterials 2012 Oct;33(30):7366-74 doi: 101016/jbiomaterials201206041 Epub 2012 Jul 29 (2012).

[34] Giraud-Guille MM, Mosser G, Belamie E. Liquid crystallinity in collagen systems in vitro and in vivo. Current Opinion in Colloid \& Interface Science (2008);13:303-13.

[35] Besseau L, Giraud-Guille M-M. Stabilization of Fluid Cholesteric Phases of Collagen to Ordered Gelated Matrices. Journal of Molecular Biology (1995);251:197-202.

[36] Giraud-Guille M-M. Liquid crystallinity in condensed type I collagen solutions: A clue to the packing of collagen in extracellular matrices. Journal of Molecular Biology (1992);224:861-73.

[37] Gobeaux F, Mosser G, Anglo A, Panine P, Davidson P, Giraud-Guille MM, et al. Fibrillogenesis in dense collagen solutions: a physicochemical study. J Mol Biol 2008 Mar 7;376(5):1509-22 doi: 101016/jjmb200712047 Epub 2008 Jan 3 (2008).

[38] Bouligan.Y. TWISTED FIBROUS ARRANGEMENTS IN BIOLOGICAL-MATERIALS AND CHOLESTERIC MESOPHASES. Tissue Cell (1972);4:189-\&.

[39] Gobeaux F, Belamie E, Mosser G, Davidson P, Panine P, Giraud-Guille MM. Cooperative Ordering of Collagen Triple Helices in the Dense State. Langmuir (2007);23:6411-7.

[40] Jee SS, Kasinath RK, DiMasi E, Kim Y-Y, Gower L. Oriented hydroxyapatite in turkey tendon mineralized via the polymer-induced liquid-precursor (PILP) process. CrystEngComm (2011);13:2077-83.

[41] Li Y, Thula TT, Jee S, Perkins SL, Aparicio C, Douglas EP, et al. Biomimetic mineralization of woven bone-like nanocomposites: role of collagen cross-links. Biomacromolecules $2012 \mathrm{Jan}$ 9;13(1):49-59 doi: 101021/bm201070g Epub 2011 Dec 1 (2012).

[42] Olszta MJ, Cheng X, Jee SS, Kumar R, Kim Y-Y, Kaufman MJ, et al. Bone structure and formation: A new perspective. Materials Science and Engineering: R: Reports (2007);58:77-116. [43] Thula-Mata T, Burwell A, Gower LB, Habeliz S, Marshall GW. Remineralization of Artificial Dentin Lesions via the Polymer-Induced Liquid-Precursor (PILP) Process. Materials Research Society symposia proceedings Materials Research Society (2011);1355:1114.

[44] Rodriguez DE, Thula-Mata T, Toro EJ, Yeh YW, Holt C, Holliday LS, et al. Multifunctional role of osteopontin in directing intrafibrillar mineralization of collagen and activation of osteoclasts. Acta Biomater (2014);10:494-507. 
[45] Nudelman F, Lausch AJ, Sommerdijk NAJM, Sone ED. In vitro models of collagen biomineralization. Journal of Structural Biology (2013);183:258-69.

[46] Zhang W, Luo X-j, Niu L-n, Yang H-y, Yiu CKY, Wang T-d, et al. Biomimetic Intrafibrillar Mineralization of Type I Collagen with Intermediate Precursors-loaded Mesoporous Carriers. Scientific Reports (2015);5:11199.

[47] Deshpande AS, Beniash E. Bio-inspired Synthesis of Mineralized Collagen Fibrils. Crystal growth \& design (2008);8:3084-90.

[48] Jee SS, Thula TT, Gower LB. Development of bone-like composites via the polymer-induced liquid-precursor (PILP) process. Part 1: influence of polymer molecular weight. Acta Biomater 2010 Sep;6(9):3676-86 doi: 101016/jactbio201003036 Epub 2010 Mar 30 (2010).

[49] Wang Y, Azais T, Robin M, Vallee A, Catania C, Legriel P, et al. The predominant role of collagen in the nucleation, growth, structure and orientation of bone apatite. Nat Mater $2012 \mathrm{Jul}$ 1;11(8):724-33 doi: 101038/nmat3362 (2012).

[50] Brodsky B, Eikenberry EF, Belbruno KC, Sterling K. Variations in collagen fibril structure in tendons. Biopolymers (1982);21:935-51.

[51] Thula TT, Rodriguez DE, Lee MH, Pendi L, Podschun J, Gower LB. In vitro mineralization of dense collagen substrates: a biomimetic approach toward the development of bone-graft materials. Acta Biomater 2011 Aug;7(8):3158-69 doi: 101016/jactbio201104014 Epub 2011 Apr 20 (2011). [52] Landis WJ, Song MJ, Leith A, McEwen L, McEwen BF. Mineral and Organic Matrix Interaction in Normally Calcifying Tendon Visualized in Three Dimensions by High-Voltage Electron Microscopic Tomography and Graphic Image Reconstruction. Journal of Structural Biology (1993);110:39-54.

[53] Nudelman F, Bomans PHH, George A, de With G, Sommerdijk NAJM. The role of the amorphous phase on the biomimetic mineralization of collagen. Faraday Discussions (2012);159:357-70.

[54] Ermanno B. The Mineralization of Bone and Its Analogies with Other Hard Tissues.

[55] Staines KA, MacRae VE, Farquharson C. The importance of the SIBLING family of proteins on skeletal mineralisation and bone remodelling. The Journal of endocrinology (2012);214:241-55. 
Figure Captions

Fig. 1. Biomimetic mineralization overview. (A) LCC scaffold prior to mineralization. (B) The same scaffold cut into pieces, mineralized for 2 weeks via the PILP process, then lyophilized. (C) SEM image of a mineralized, aligned lamella and corresponding EDS (inset). (D) Unmineralized LCC scaffold stained to enhance contrast. Note - the dark region in the bottom left corner is an artifact of the staining process. (E) After 2 week PILP mineralization, a similar area of aligned collagen appears to have templated the intrafibrillar mineral organization. (F) XRD pattern of PILP-mineralized LCC, with comparison to bovine femur. (G) TGA ash weight measurements after combustion indicate the scaffold comprised $\sim 70$ wt. $\%$ mineral.

Fig. 2. Liquid Crystalline Collagen (LCC) scaffolds. (A) PLM birefringence patterns (identified by the asterisks and double-headed arrow) from a semi-thin $(\sim 1 \mu \mathrm{m})$ section of aligned collagen taken with a gypsum waveplate, and without (inset), to examine liquidcrystalline texture. The waveplate is positioned at $45^{\circ}$ to the crossed-polars, therefore collagen fibril axes which are parallel to the waveplate appear bright blue while those oriented perpendicular appear orange. (B) SEM image of unmineralized LCC interior; EDS (inset) shows a lack of mineral signal, as expected. (C) Low-mag. TEM image of unstained, unmineralized LCC cross-section. The dark lines running across the sample are folds in the section, artifacts from sample preparation (D) Area of interest from C, with brightness and contrast enhanced to help visualize fibril orientation and banding patterns. $(\mathbf{E} \&$ F) Heavy metal staining was also used to enhance contrast, where it aided in visualization of other organizational patterns that were present, including rotating alignment (E) and isotropic domains (F) of banded fibrils. In some instances, the Dbanding remained in registry across multiple fibrils (arrows).

Fig. 3. PILP-mineralized LCC scaffolds. (A) Low-mag. SEM micrograph of LCC interior after the surface lamella delaminated during sample preparation. The EDS spectrum (inset right) indicates high mineral content (large $C a$ and $P$ peaks relative to $C$ peak), although mineral crystals cannot be seen even at high magnification (inset top right). (B) SEM image showing the fibrillar, cholesteric organization in the interior of a freeze-dried, mechanically fractured specimen. (C) Irregular, thin hydroxyapatite mineral platelets mechanically extracted from a pulverized sample. Arrows indicate platelets viewed edge-on, which appear as dark streaks (black arrows) in bright field TEM. (D) Mineralized collagen 'particle' obtained from a pulverized specimen with almost orthogonal overlapping collagen fibrils; note the dark streaks well-aligned with the fibril axes. (E) Dark $67 \mathrm{~nm}$ banding can be seen in register across two or more parallel fibrils. (F) Higher magnification of a thinner edge from $E$ showing the mineral co-oriented with the fibril axis (regions between arrows).

Fig. 4. Visualization of nanocrystal orientation in a parallel array of collagen fibrils. (A \& B) Bright-field TEM images of aligned collagen fibrils and the corresponding mineral orientation (dark streaks). (C \& D) SAED patterns generated from the areas indicated by the white circles in A and B. ( $E$ \& F) Corresponding dark-field TEM images created by selecting the (002) arc/spot indicated by the red dashed circles in $\mathrm{C}$ and $\mathrm{D}$; crystals contributing to the selected beam appear bright against a dark background. 
Fig. 5. XRD of PILP-mineralized LCC from four separate experiments to show reproducibility. The sharp peaks around $26.5^{\circ}$ and $53^{\circ}$ result from diffraction of the (002) and (004), respectively. The broad peak around $32^{\circ}$ is composed of overlapping peaks from the (211), (112), and the (300) planes.

Fig. 6. TGA of PILP-mineralized LCC from four separate mineralizations to show reproducibility. Dense collagen scaffolds were mineralized via the PILP process with 50 $\mu \mathrm{g} / \mathrm{ml}$ of $27 \mathrm{kDa}$ pAsp for two weeks. The scaffolds contained a mineral content of similar degree to that of native bone (60-70 wt. \%). 
Figure 1
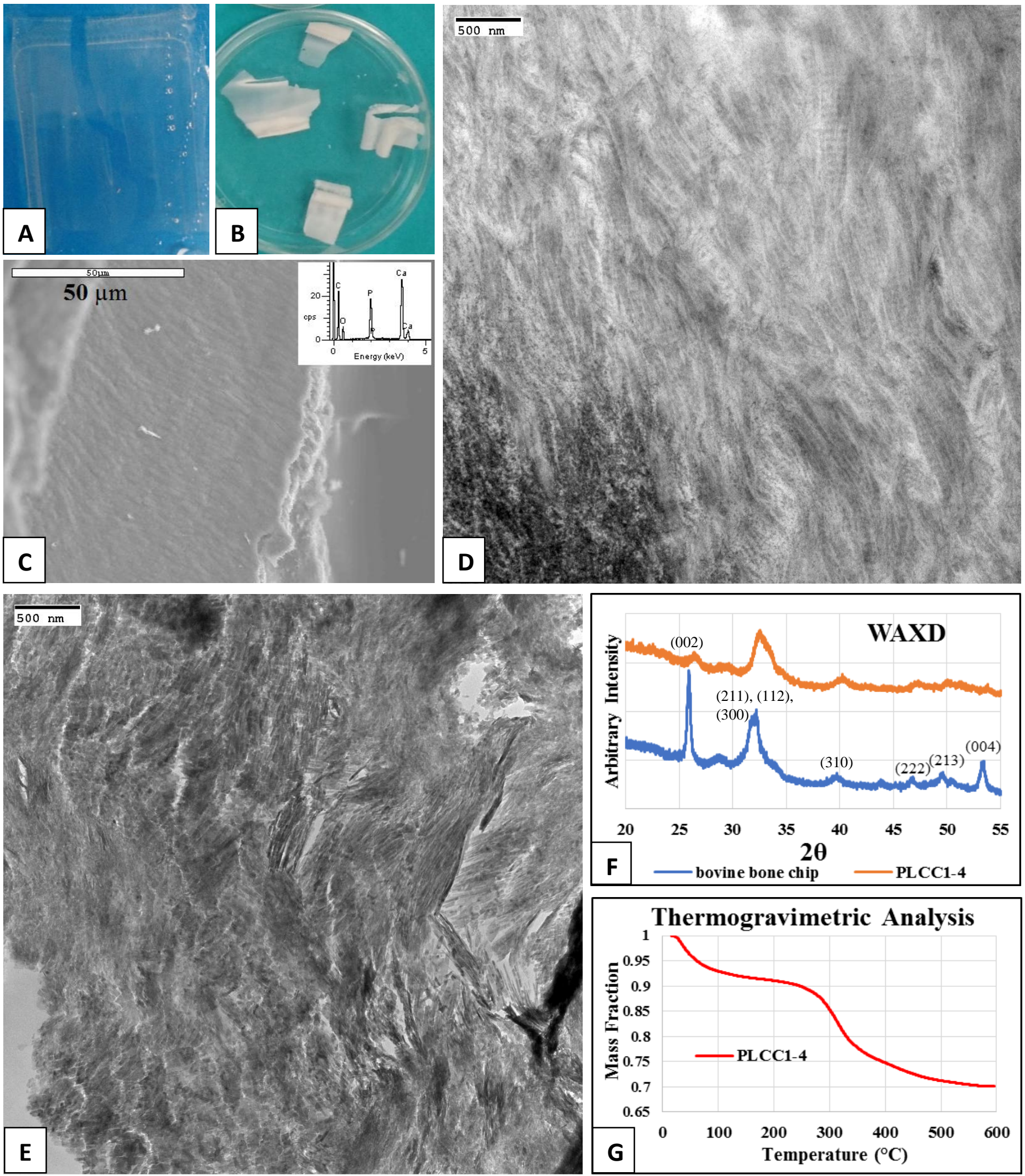
Figure 2
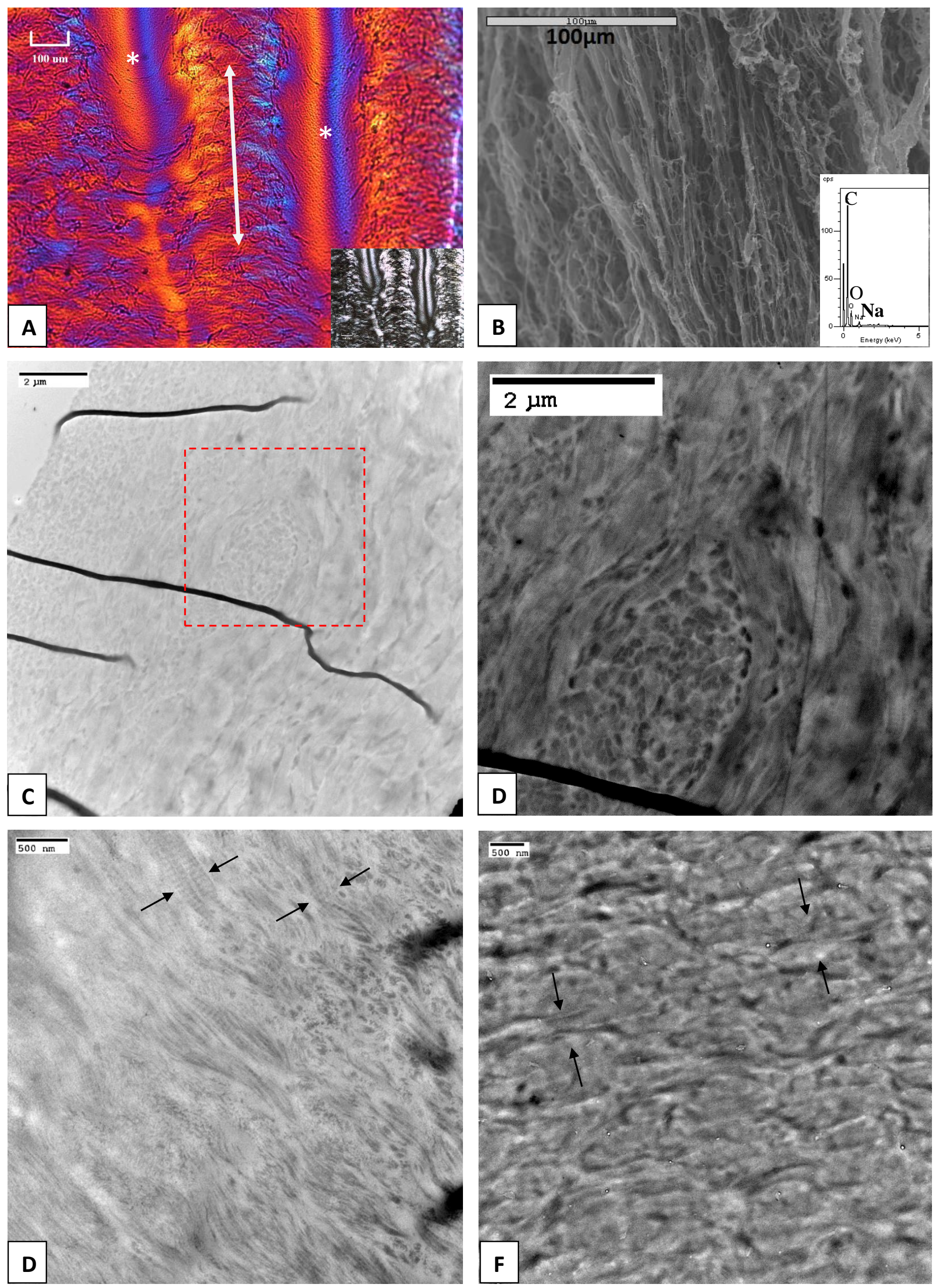
Figure 3
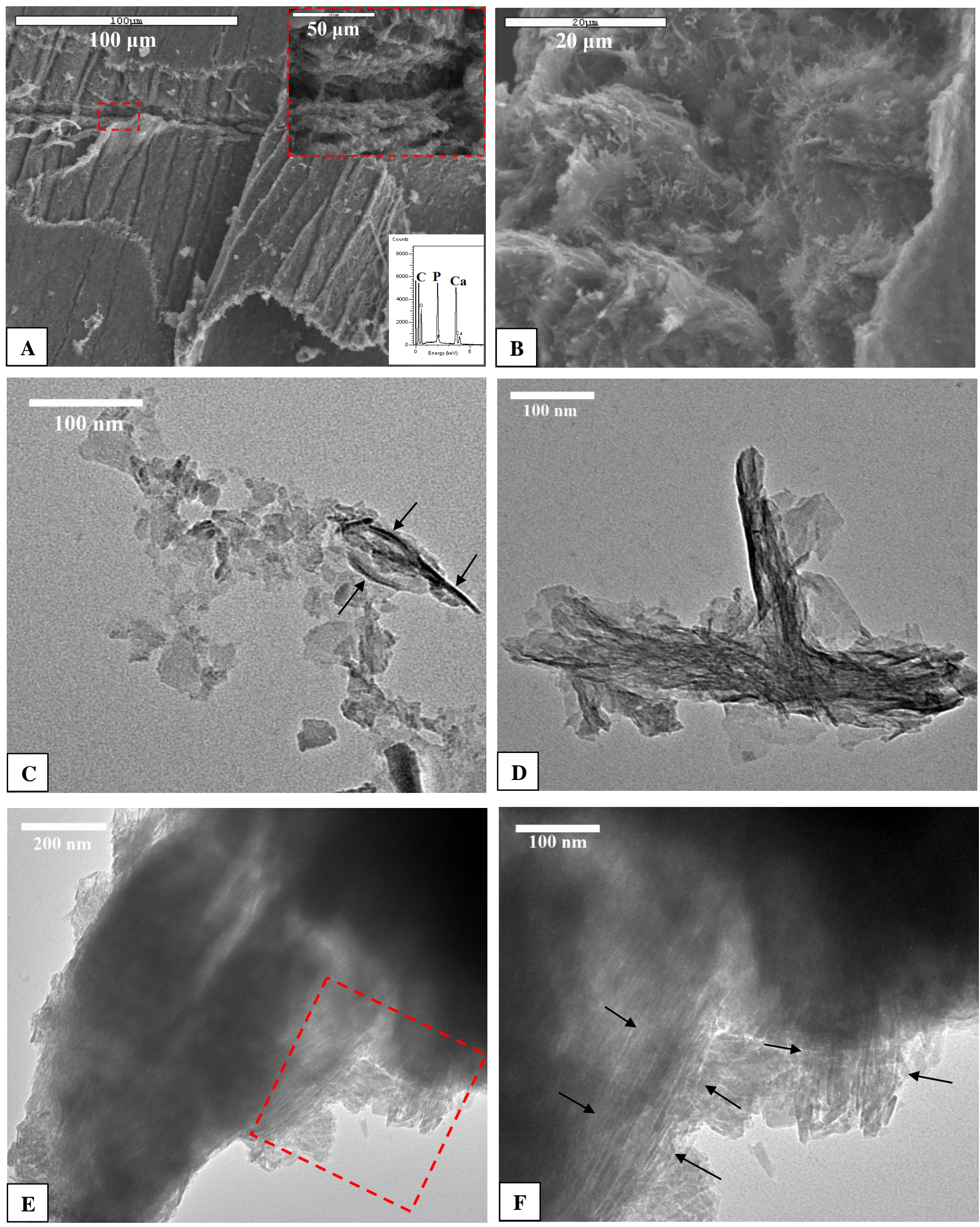
Figure 4
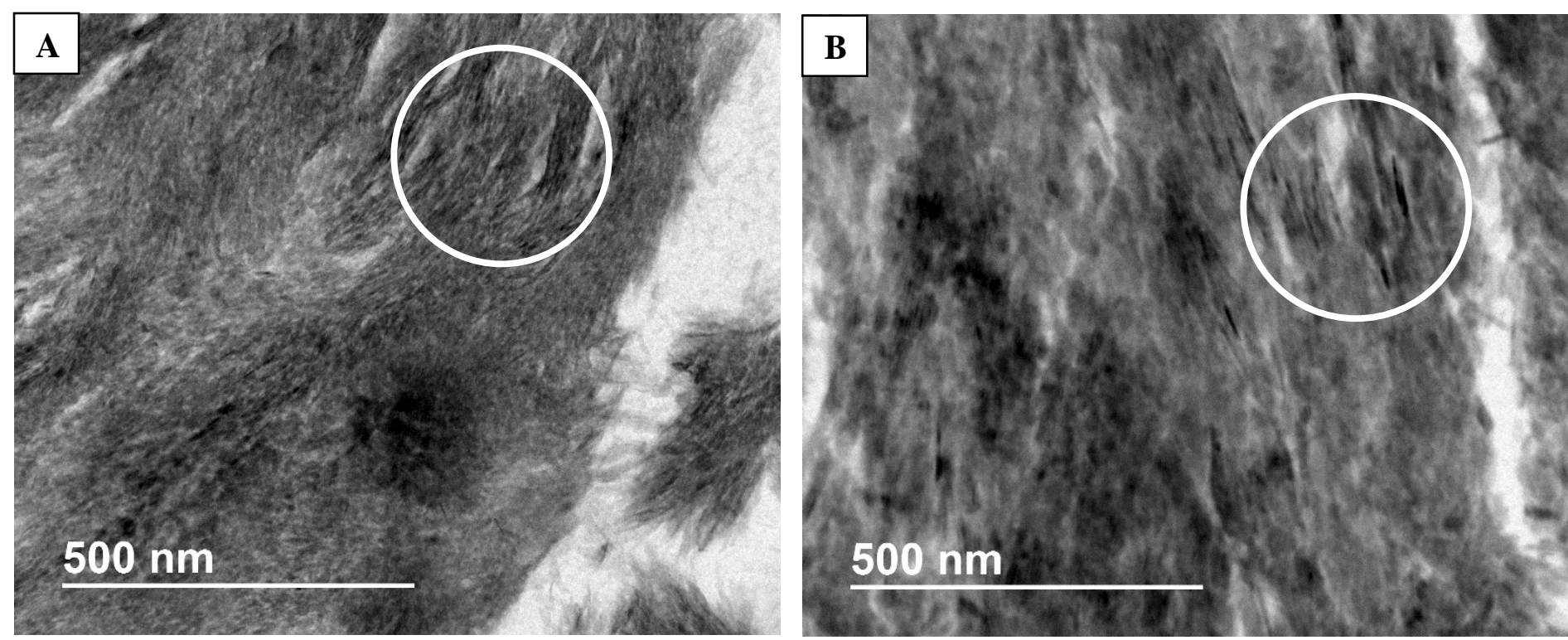

C

$101 / \mathrm{nm}$

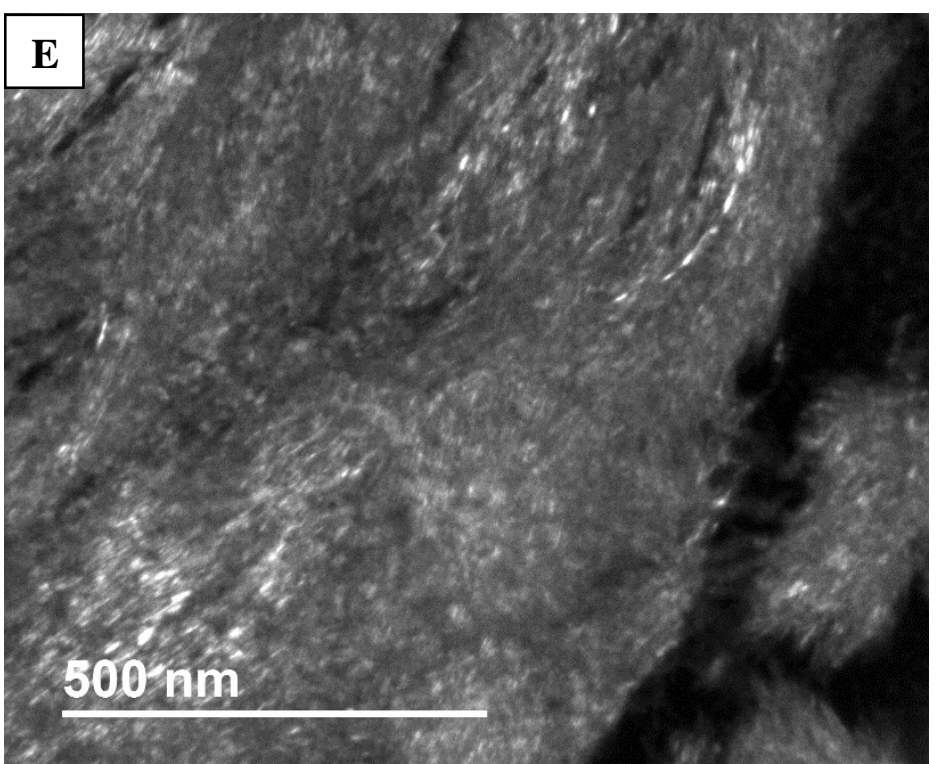

D

$101 / \mathrm{nm}$

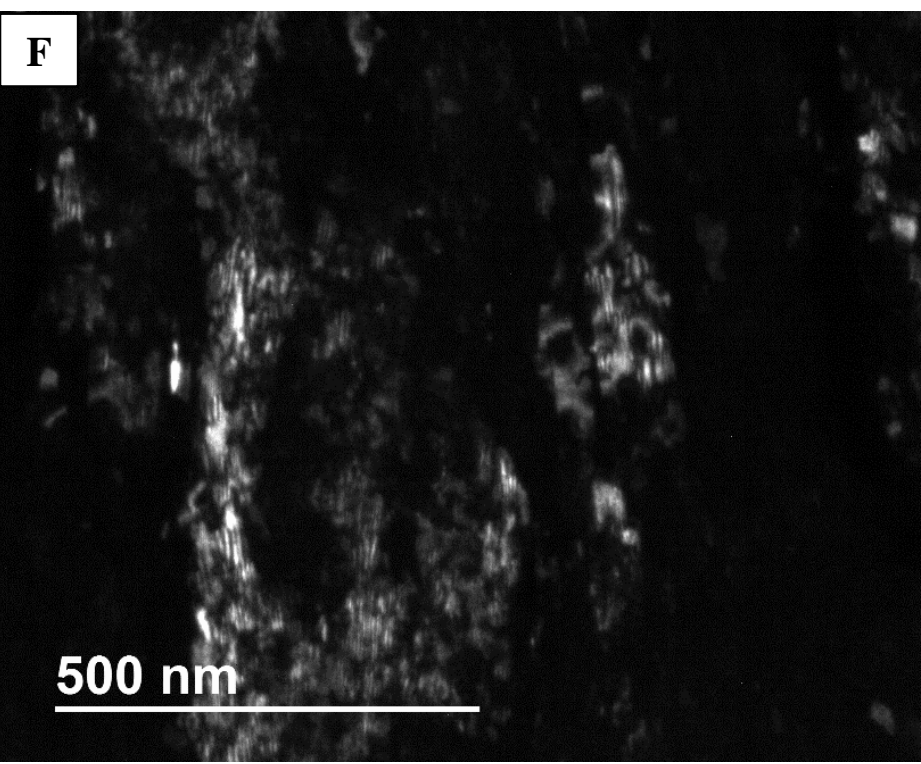


Figure 5

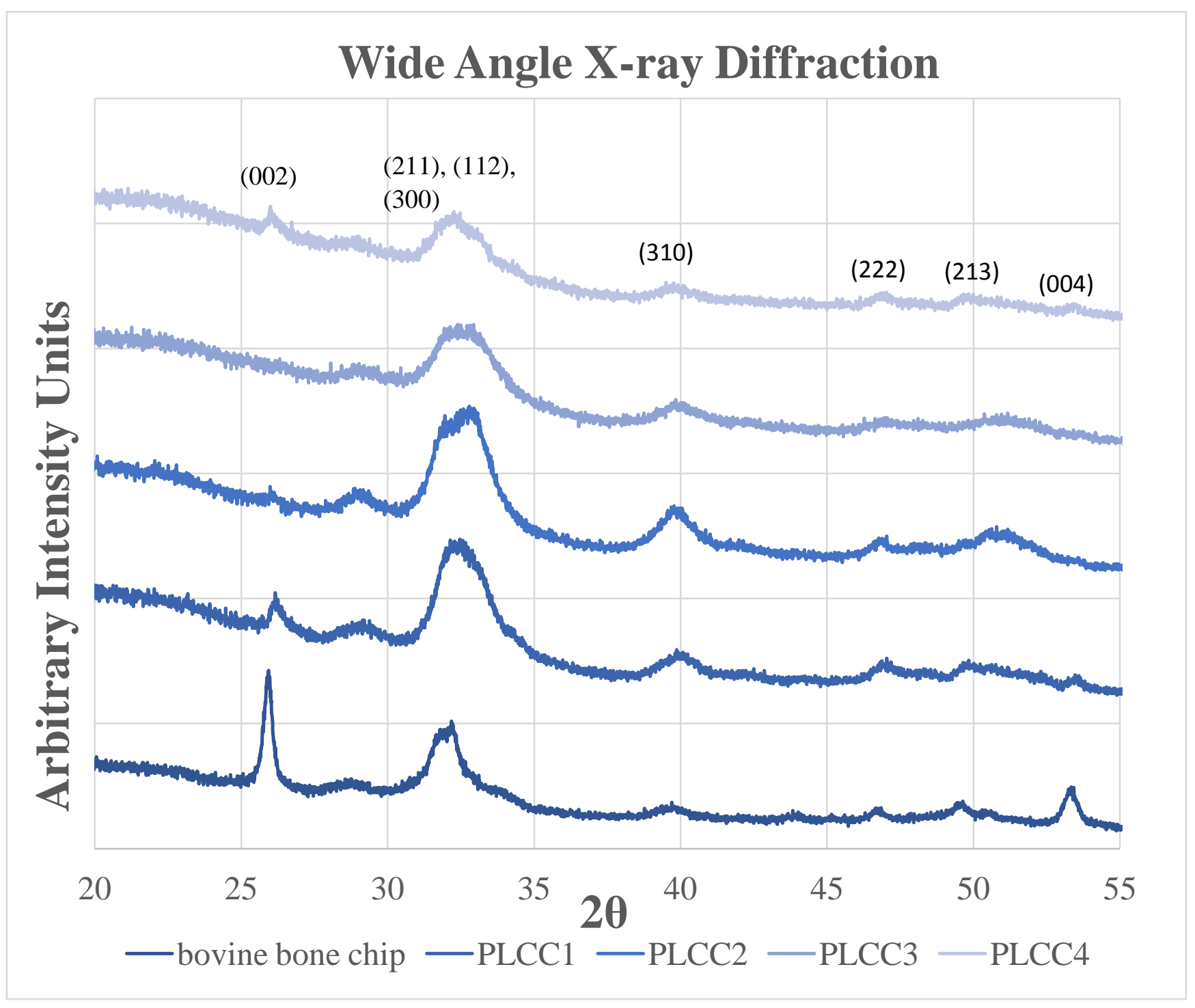


Figure 6

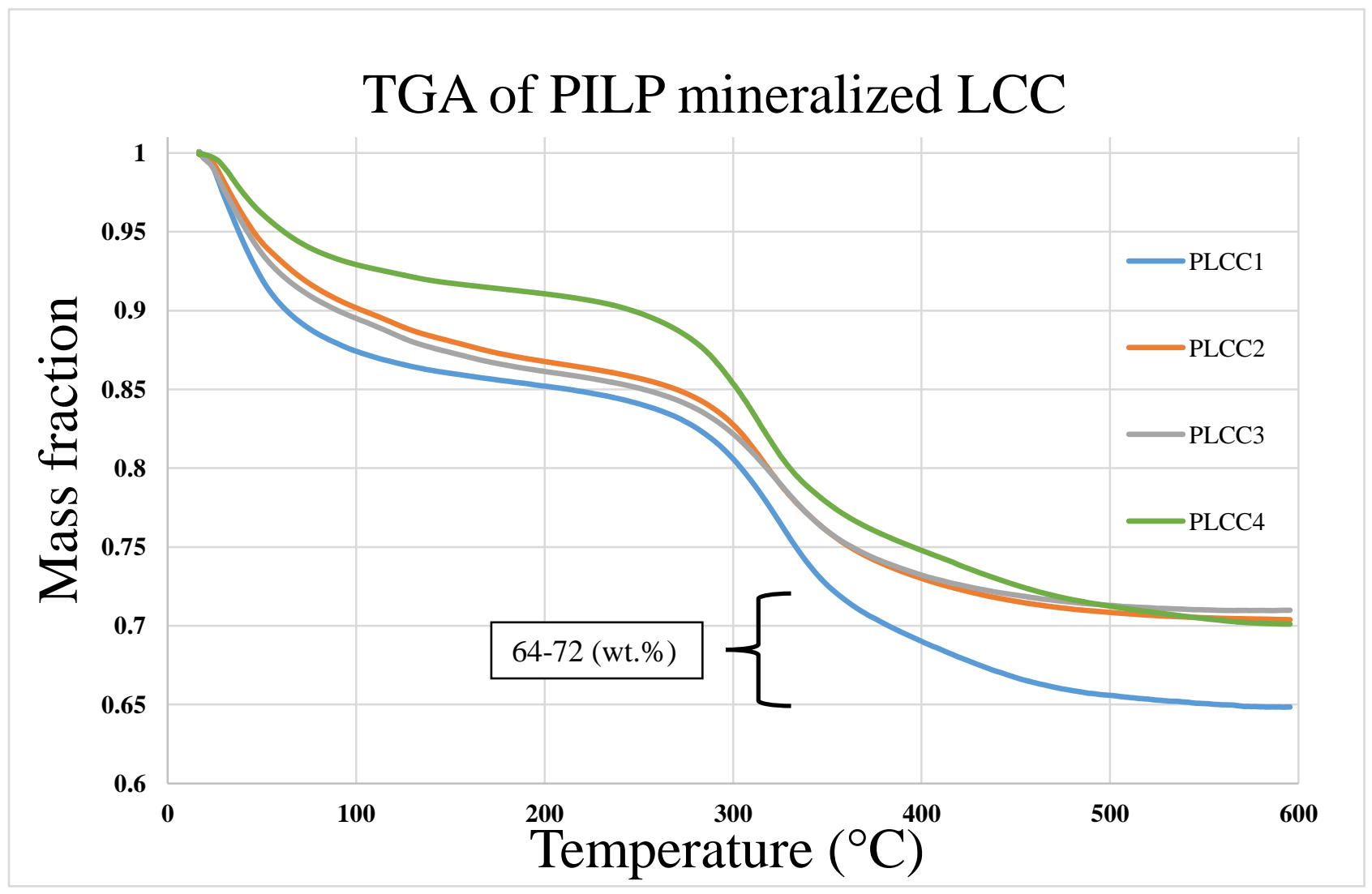

